\title{
Increased alpha-taxilin protein expression is associated with the metastatic and invasive potential of renal cell cancer
}

\author{
Tomoko Mashidori $^{1 *}$, Hiromichi Shirataki ${ }^{2 *}$, Takao Kamai $^{*}$, Fumihiko NaKamura ${ }^{1}$, and Ken-Ichiro Yoshida ${ }^{1}$ \\ ${ }^{1}$ Department of Urology, Dokkyo Medical University, Mibu, Tochigi, Japan and ${ }^{2}$ Department of Molecular and Cell Biology, Dokkyo \\ Medical University, Mibu, Tochigi, Japan
}

(Received 8 November 2010; and accepted 20 December 2010)

\begin{abstract}
Intracellular vesicle trafficking is the principal transportation system in eukaryotic cells, and is considered to be involved in a variety of processes related to cell proliferation. A protein named alpha-taxilin has been identified as a binding partner of the syntaxin family, which coordinates intracellular vesicle trafficking. To clarify the role of alpha-taxilin in renal cell carcinoma (RCC), we investigated alpha-taxilin protein expression in clear cell RCC tissues. We analyzed alphataxilin protein in matched sets of tumor and non-tumor tissues from the surgical specimens of 52 Japanese RCC patients by Western blotting. We also studied the relation between alpha-taxilin protein expression in tumor tissues and various clinicopathological features. The alpha-taxilin protein level was higher in tumor tissues than in non-tumor tissues $(P<0.05)$. Increased expression of alpha-taxilin protein in primary tumors was related to local invasion $(P<0.001)$, pathological vessel invasion $(P<0.001)$, and metastasis $(P<0.0001)$. Kaplan-Meier plots of survival for patients with low versus high alpha-taxilin expression revealed that high expression in tumor tissues was associated with shorter overall survival in all patients $(P<0.05)$ and with shorter disease-free survival in patients without metastasis $(P<0.01)$. These findings suggest that alpha-taxilin influences the metastatic and invasive potential of RCC.
\end{abstract}

Intracellular vesicle trafficking is the principal transportation system in eukaryotic cells, and vesicle trafficking is thought to be involved in various processes related to cell proliferation. Abnormal proliferation is the hallmark of cancer. Recently, a protein named alpha $(\alpha)$-taxilin was identified as a binding partner of the syntaxin family, which is involved in the coordination of intracellular vesicle trafficking (26). The taxilin family has at least three members - $\alpha$-, beta $(\beta)$-, and gamma $(\gamma)$-taxilin-and $\alpha$-taxilin possesses an extremely long coiled-coil region and interacts with syntaxin family members localized on the plasma membrane, but not with those

Address correspondence to: Takao Kamai M.D. Department of Urology, Dokkyo Medical University, 880 Kitakobayashi Mibu-machi, Tochigi 321-0293, Japan Tel: +81-282-87-2162, Fax: +81-282-86-7533

E-mail: kamait@dokkyomed.ac.jp localized on intracellular organelles $(26,28)$. There is evidence that the taxilin family is involved in the transfer of vesicles delivered to the plasma membrane $(26,28)$. However, the precise influence of the taxilin family on the functions of the syntaxin family remains to be fully elucidated, and the actual role of $\alpha$-taxilin in intracellular vesicle trafficking is also unknown. In addition to providing information about the molecular mechanisms involved, examination of the physiological and pathophysiological functions of taxilin should improve our understanding about the role of this family of proteins.

We recently reported that $\alpha$-taxilin protein is significantly upregulated in proliferating neural stem cells during embryonic development in the rat (37). In addition, Oba-Shinjo et al. recently reported that

\footnotetext{
* These authors contributed equally to this work.
} 
$\alpha$-taxilin mRNA is upregulated in astrocytic tumor cells compared with non-tumorous central nervous system cells, indicating that $\alpha$-taxilin may be related to tumor development (29). Thus, it is possible that $\alpha$-taxilin is involved in tumorigenesis through modulation of cell proliferation and other processes. However, previous studies have examined the expression of $\alpha$-taxilin mRNA, but not $\alpha$-taxilin protein, and have not assessed the detailed relationship between the level of $\alpha$-taxilin protein expression and various clinicopathological features. To determine whether $\alpha$-taxilin protein is indeed upregulated and to clarify the clinical significance of $\alpha$-taxilin protein expression in tumors seem to be important objectives. In addition to providing information on molecular mechanisms, addressing these issues will improve our understanding of the functions of the taxilin family. However, the accumulating evidence were obtained in cell culture studies, so it is also important to investigate the role of $\alpha$-taxilin in human cancer tissues.

Localized renal cell carcinoma (RCC) is generally considered to be indicated for surgical treatment, but almost $30 \%$ of the patients with limited disease at the time of surgery develop metastasis within the next 3 years (1). The prevalence of RCC is steadily increasing, and it now accounts for $2-3 \%$ of all adults malignancies. About $40 \%$ of RCC patients die of metastatic disease, because of the high frequency of metastasis at the time of diagnosis or relapse following nephrectomy (23). RCC patients with distant metastases have a poor prognosis and their 5-year survival rate is less than $10 \%$ (1). Clear cell RCC is considered to be an immunogenic tumor (13) and cytokine therapy is the mainstay of treatment (5), since this cancer is notoriously resistant to chemotherapy or radiotherapy (23).

Because clear cell RCC is an extremely vascular tumor, a high percentage of patients already have metastasis at the time of diagnosis (23). It has been reported that vascular endothelial growth factor (VEGF) expression is upregulated in RCC and that hematogenous metastasis occurs early, leading to an unfavorable prognosis (21). A pathway that includes the von Hipple-Lindau (VHL) tumor suppressor gene, hypoxia-inducible factor-1 (HIF-1) alpha, and VEGF is important for metastasis of clear cell RCC, and targeting this pathway may improve the prognosis of patients with metastatic RCC (38). Inhibition of metastasis could be a promising treatment strategy for advanced RCC, but many of the molecular mechanisms involved in invasion and metastasis by this cancer are yet to be elucidated.
Cell migration is important for tumor metastasis $(9,32)$, and vesicle trafficking is widely accepted to be essential for cell migration $(14,24)$. There is also increasing evidence that vesicle-mediated protein transportation has an important role in tumorigenesis, angiogenesis, tumor invasion and metastasis, and tumor immune privilege (43). Taken together, these observations suggest that vesicle trafficking may be associated with the progression and metastasis of human tumors. However, we have no data regarding the role of vesicle trafficking in RCC. To address the question of whether molecules related to vesicle trafficking are involved in the development and progression of RCC, we compared the expression of $\alpha$-taxilin protein - the first identified and best-characterized of these molecules - in RCC tissues with that in corresponding non-tumor tissues from nephrectomy specimens by Western blotting. The relation between expression of $\alpha$-taxilin and various clinicopathological features was also examined. Furthermore, we assessed the usefulness of $\alpha$-taxilin for predicting the survival of patients with clear cell RCC. The results of this study might provide new insights into the role of taxilin in human cancers.

\section{MATERIALS AND METHODS}

Patients and tissue specimens. We studied 52 consecutive Japanese patients ( 37 men and 15 women) aged 35 to 77 years (mean age: 62.9 years) who were diagnosed as having clear cell RCC (with no sarcomatoid or rhabdoid components) from 2004 to 2009. All patients underwent imaging with CT and/or MRI for preoperative staging prior to radical nephrectomy. The postoperative follow-up period ranged from 2 to 68 months (median: 29 months). Patients underwent surgery before receiving any other therapy.

In each patient, three different tumor sites and various parts of the non-neoplastic kidney were harvested for this study. The resected tissues were stored at $-80^{\circ} \mathrm{C}$, as described previously $(15,41)$. The tumor grade and clinical stage were determined according to the Fuhrman grading system and the TNM classification, respectively $(8,39)$. In the present study, all of the tumors were histological grade 1 to 3 . This study was conducted in accordance with the Declaration of Helsinki and the institutional review board of Dokkyo Medical University Hospital gave its approval. In addition, each patient signed a consent form approved by the Committee on Human Rights in Research of our institution. 
Postoperative immunotherapy with interferon (IFN)-alpha $(3,5$, or 6 million units of natural human IFN-alpha two or three times a week) was given to ten patients, while sorafenib (400 or $800 \mathrm{mg}$ /day) was administered to three patients and sunitinib ( 25 to $50 \mathrm{mg}$ /day for 4 weeks, followed by two weeks of rest) was used in two patients. Therapy was usually administered to patients with extra-renal involvement (metastatic disease) until progression occurred. The doses of these agents were decreased if grade $3 / 4$ toxicity occurred.

The tumor tissues and normal tissues of the resected specimens were carefully dissected free of stromal elements. Western blotting was carried out as described previously (15). Briefly, $50 \mu \mathrm{g}$ of cytosolic protein was separated by SDS-PAGE $(12.5 \% \mathrm{gel})$ and electrotransferred to a polyvinylidene difluoride membrane (Immobilon-P membranes; Millipore, Bedford, MA). After the membrane was blocked, the bound proteins were probed with a specific antibody for $\alpha$-taxilin $(26,28)$. Hela cells were used as a positive control for $\alpha$-taxilin expression. Then the membrane was washed and incubated with horseradish peroxidase-conjugated secondary antibodies, after which protein bands were visualized by chemiluminescence. The blotted membrane was scanned for densitometry with a PDI imaging scanner (Agfa Japan, Tokyo) and data were analyzed with NIH Image software. The level of $\alpha$-taxilin protein expression was calculated as a ratio to that of beta actin in both tumor tissue and the corresponding normal tissue (mean \pm S.D.). Since interindividual variations in the expression of $\alpha$-taxilin may be important, for quantification of protein levels, the relative amount of $\alpha$-taxilin in each sample of tumor tissue was expressed as a ratio of the amount in the corresponding normal tissue sample (which was set at 1.0) by densitometric analysis of the bands from both specimens, as described previously $(7,15)$. Mean values for tumor tissues and non-tumor tissues were calculated from three experiments (15).

Cell culture. $\alpha$-Taxilin proteins expression was examined in RCC cell lines (26-28). Three human renal cancer cell lines (293, Caki-1, and TUHR4TKB) were obtained from the Riken Bioresource Cancer Cell Bank (Tsukuba, Japan). Cells were grown in RPMI-1640 medium supplemented with fetal bovine serum, $2 \mathrm{mM}$ L-glutamine, $100 \mu \mathrm{g} / \mathrm{mL}$ streptomycin, and 100 units $/ \mathrm{mL}$ penicillin (all from Life Technologies, Carlsbad, $\mathrm{CA}$ ) at $37^{\circ} \mathrm{C}$ in a humidified atmosphere with $5 \% \mathrm{CO}_{2}$. Adherent cells were detached from the culture dishes with trypsin/EDTA (Sigma, Deisenhofen, Germany).

Immunohistochemistry. Using the same antibody for $\alpha$-taxilin as that employed for Western blotting, immunohistochemistry was done on 5 tumors, as described previously (15), in order to confirm the data obtained by Western blotting.

Statistical analysis. Since the data did not show a normal distribution, results were analyzed by employing the non-parametric Mann-Whitney $U$ test for comparisons between two groups and the nonparametric Kruskal-Wallis test to compare three groups. Bonferroni's correction is generally employed for multiple comparisons, and the MannWhitney $U$ test was corrected by this method. Expression of $\alpha$-taxilin protein in the primary tumor, tumor grade, local stage (pT), pathological vessel invasion (VI), and metastasis (M) were all assessed for their influence on disease-free survival and overall survival by the Cox proportional hazards model using univariate and multivariate analyses. The Kaplan-Meier method was employed to estimate survival for various groups, and differences of survival were assessed by the log-rank test. In all analyses, a probability $(P)$ value of less than 0.05 was considered significant. Data were analyzed by using SPSS statistical software (version 15.0 for Windows SPSS).

\section{RESULTS}

Alpha-taxilin protein level and tumor characteristics Although expression of $\alpha$-taxilin protein was detected in both tumor tissues and non-tumor tissues, this protein was abundantly expressed in tumor tissues using Western blotting (Fig. 1). In immunohistochemistry, the cytoplasm but not the membrane of some tumor cells were positively stained using anti$\alpha$-taxilin antibody. In contrast, non-tumor cells showed negative to very weak reaction (Fig. 2). The level of this protein was higher in tumor tissues than in non-tumor tissues, and it was significantly higher in primary tumors associated with metastasis (M1) than in those without metastasis (M0) or the non-tumor tissues (M1: $4.34 \pm 2.67, \mathrm{M} 0: 1.44 \pm 0.97$ versus 1.0 for non-tumor tissues, Fig. 3A). Expression of $\alpha$-taxilin protein in the primary tumors was not related to histological grade (grade 1: $3.51 \pm$ 4.37, grade 2: $1.59 \pm 1.35$, grade 3: $2.68 \pm 1.91$, $P=0.3023$, Fig. 3B). However, higher expression of $\alpha$-taxilin was associated with local invasion (pT1-2: 


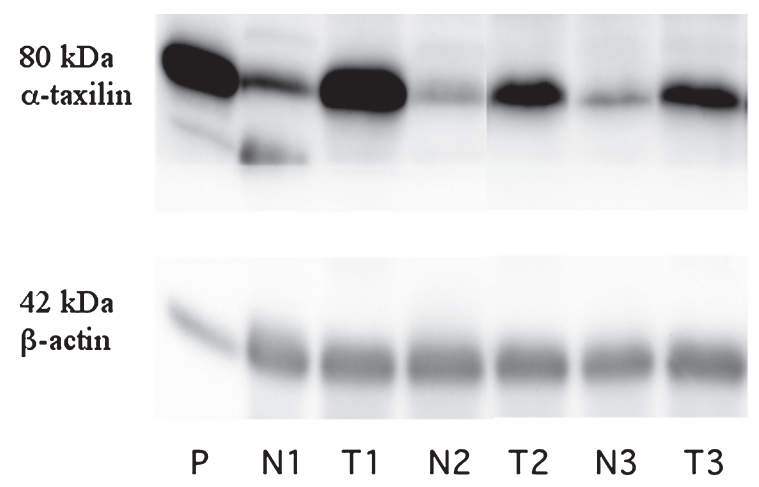

Fig. $1 a$-taxilin protein expression $(80 \mathrm{kDa})$ by Western blotting. M: Marker, P: Hela cells as positive control, T: tumor tissue, N: non-tumor tissue. Each number corresponds to a case number.

$1.33 \pm 1.13$, pT3-4: $3.57 \pm 2.85, P=0.0002$, Fig. 3 C), as well as with pathological vessel invasion $\mathrm{VI}(-)$ : $1.25 \pm 1.11, \mathrm{VI}(+): 3.47 \pm 2.72, P=0.0005$, Fig. 3D).

Alpha-taxilin and syntaxin-4 protein expression in cell lines

The order of $\alpha$-taxilin protein expression was 293 cells $>$ Caki-1 cells $>$ TUHR4TKB cells (Fig. 4 ).

\section{Alpha-taxilin protein and survival}

The mean level of $\alpha$-taxilin protein expression in tumor tissues was $2.10( \pm 1.77)$, so the patients were divided into a high expression group $(\mathrm{n}=19)$ and a low expression group $(\mathrm{n}=33)$ at this cut-off value according to the method described previously (15, 41). Kaplan-Meier plots of survival for patients with low versus high tumor expression of $\alpha$-taxilin protein showed that high expression was associated with shorter overall survival $(P=0.0390$, Fig. 5A). Univariate analysis of overall survival with the Cox proportional hazards model revealed that pathological vessel invasion and metastasis were significant variables, but multivariate analysis failed to identify any independent determinants of overall survival (Table 1).

To assess the influence on disease-free survival, patients without distant metastasis at the time of nephrectomy (M0; $\mathrm{n}=37$ ) were also divided into two groups with $\alpha$-taxilin protein levels above or below the median value of $1.24( \pm 1.00)$. In these patients with localized tumors, Kaplan-Meier analysis showed that higher $\alpha$-taxilin protein expression was related to shorter disease-free survival (Fig. 5B). The level of $\alpha$-taxilin protein expression had a significant influence according to univariate analysis by the Cox proportional hazards model, but this was

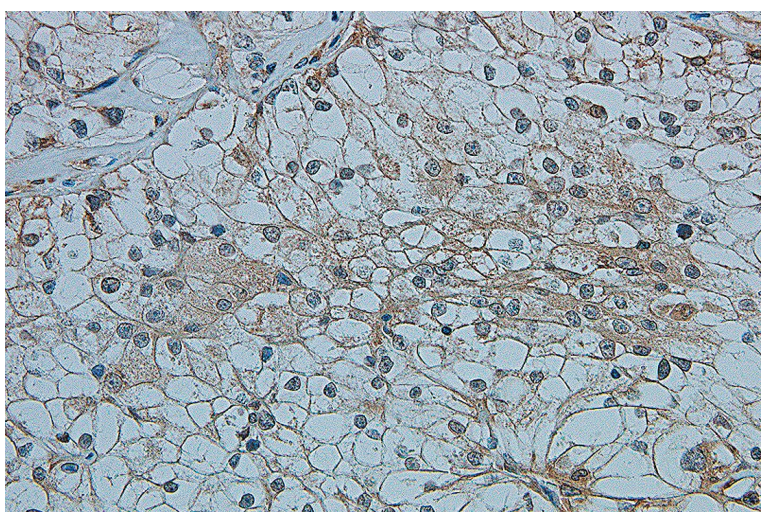

Fig. 2 Immunohistochemistry for $a$-taxilin proteins showing a brown staining in the cytoplasm of clear cell renal cell carcinoma $(\times 100)$.

not confirmed by multivariate analysis (Table 1$)$.

\section{DISCUSSION}

To allow for possible inter-individual variation of $\alpha$-taxilin protein expression in human RCC tissues, we compared $\alpha$-taxilin protein expression between paired samples of tumor and non-tumor tissues from the same kidney. Our findings showed that $\alpha$-taxilin protein levels were higher in tumor tissues than in non-tumor tissues, and were significantly higher in the primary tumors with metastasis (M1) than in those without metastasis (M0). Furthermore, higher expression of $\alpha$-taxilin protein in the primary tumors was correlated with local invasion, pathological vessel invasion, and an unfavorable prognosis. $\alpha$-Taxilin protein showed different levels of expression in three cultured cell lines, indicating that the different subtypes of human RCCs have different $\alpha$-taxilin expression patterns. To our knowledge, this is the first investigation of $\alpha$-taxilin in RCC, and the findings suggest that it may be involved in the metastatic and invasive potential of this cancer.

\section{Tumor invasion and metastasis}

In the present study, higher $\alpha$-taxilin protein levels were associated with local invasion, pathological vessel invasion, and metastasis, and high $\alpha$-taxilin expression was associated with shorter overall survival. Local invasion and pathological vessel invasion had a significant influence on overall survival according to Cox univariate analysis. Furthermore, in patients who were M0 at nephrectomy, $\alpha$-taxilin protein expression was associated with local invasion and pathological vessel invasion, and high $\alpha$-taxilin expression was associated with early me- 


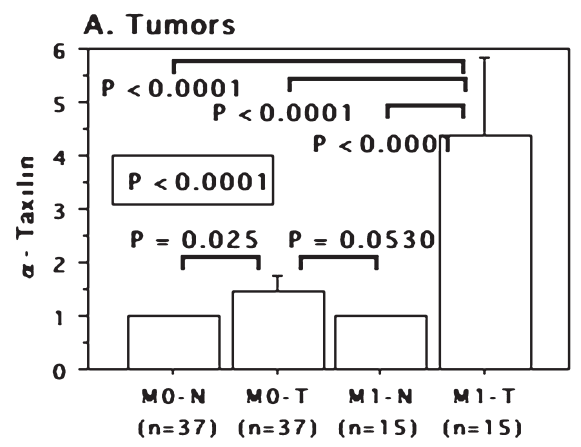

C. pT stage

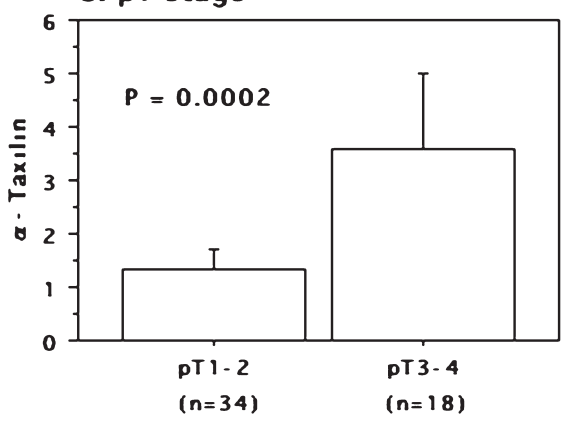

B. Grade

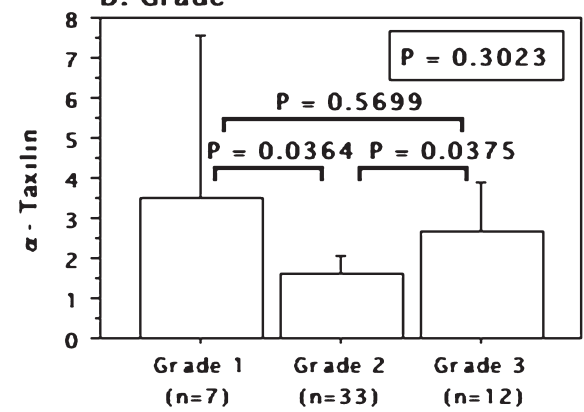

D. Pathological vessel invasion

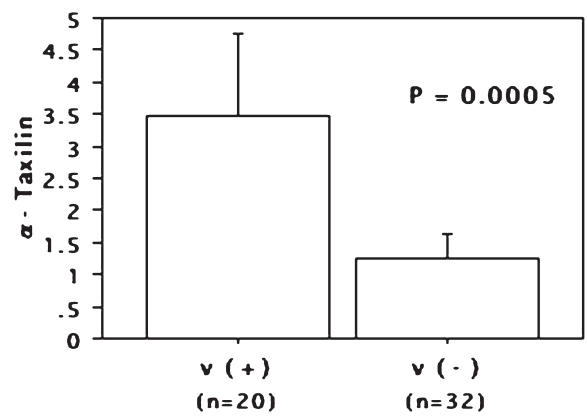

Fig. 3 The relative expression levels of $a$-taxilin protein in tumor to those in corresponding non-tumor portion, which was set to 1.0. A; Expression in primary tumor with metastasis (M1-T) and its corresponding non-tumor tissues (M1-N), and non-metastatic primary tumor (M0-T) and its corresponding non-tumor tissues (M0-N). Squared $P$ values were obtained by comparing the four groups with the Kruskal-Wallis test. B; Expression in Grade. Squared $P$ values were obtained by comparing the three groups with the Kruskal-Wallis test. C; Expression in pT stage. D; Expression in pathological vessel invasion. The data show the $95 \%$ confidential interval.

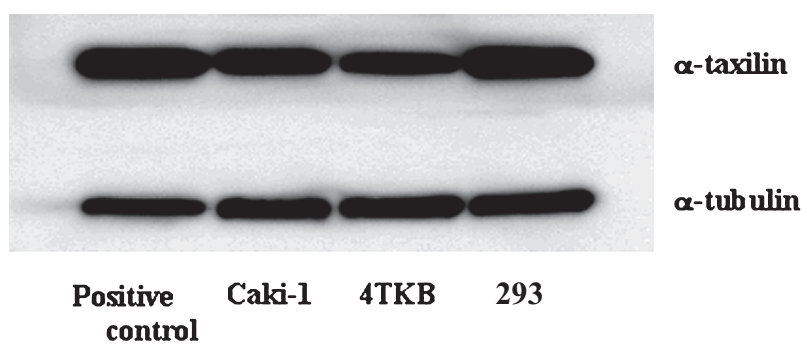

Fig. 4 The expression of $a$-taxilin proteins in the human renal cancer cell lines (Caki-1, TUHR4TKB, and 293) by Western blotting. Hela cells were used as positive control.

tastasis. These findings suggested that $\alpha$-taxilin is associated with the metastatic and invasive potential of RCC. Metastasis and invasion involve a complex series of processes that include cell adhesion, proteolysis of the extracellular matrix by matrix metalloproteinases (MMPs), and migration of tumor cells (40). It has been reported that overexpression of epimorphin/syntaxin-2 in the mouse mammary gland is related to carcinogenesis (2). Miyata et al. reported that syntaxin-4 is involved in the intracellular trans- port of membrane-type 1 matrix metalloproteinase (MT1-MMP), which plays multiple roles in tumor cell invasion and metastasis, to the plasma membrane in human gastric epithelial cells (22). In addition, a dominant-negative mutant form of syntaxin-4 is associated with reduced expression of MT1-MMP on the plasma membrane and inhibition of cell invasion. Moreover, it has been reported that soluble- $N$ ethylmaleimide-sensitive factor attachment protein receptor (SNARE) is involved in the secretion of MMP-2 and MMP-9, translocation of MT1-MMP to the plasma membrane, degradation of the extracellular matrix, and cell invasion (18). MMP-9 has a key role in promoting angiogenesis and metastasis (3), and higher expression of MMP-2 and MMP-9 proteins is associated with an unfavorable prognosis for RCC patients (17). Therefore, it is possible that $\alpha$-taxilin is involved in intracellular transport of MMPs in RCCs through an interaction with syntaxin family members.

\section{Vesicle trafficking}

We recently identified $\alpha$-taxilin as a novel syntaxin- 
binding protein involved in intracellular vesicle trafficking (26). The process of vesicle trafficking is important for intracellular signaling and protein

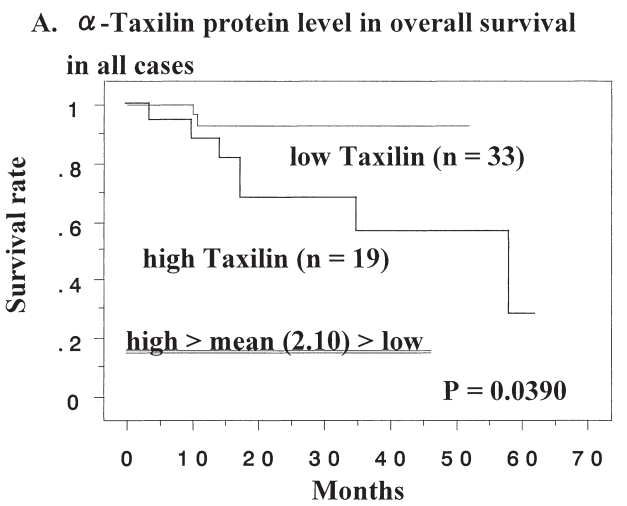

B. $\alpha$-Taxilin protein level in disease-free survival in M0 cases at nephrectomy

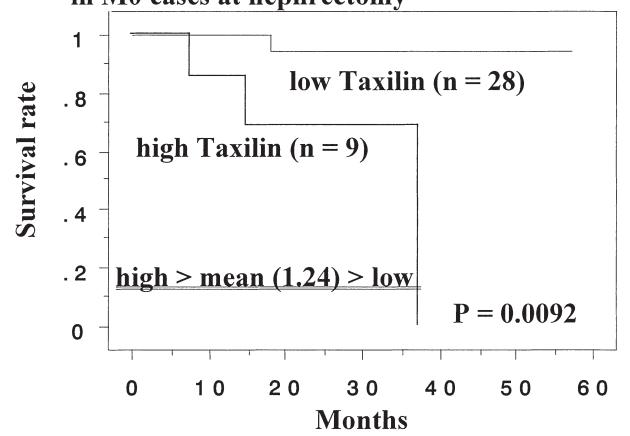

Fig. 5 Overall survival curve (A) in all patients based on the mean values of $a$-taxilin protein expression (2.10) in tumor tissues, and disease-free survival curve (B) in M0 patients at nephrectomy based on the mean values of $\alpha$-taxilin protein expression (1.24) in tumor tissues; the cases were divided into two groups at this levels-high and low expression. targeting, and is also widely considered to be essential for cell migration $(14,24)$, which in turn is required for tumor metastasis $(9,32)$. There is also increasing evidence that vesicle trafficking has an important role in tumorigenesis, angiogenesis, invasion, metastasis, and tumor immune privilege (43). Intracellular membrane trafficking consists of a series of coordinated steps that result in vesicular transport of cargo proteins to specific locations within the cell. Most trafficking is mediated by SNAREs located on the vesicle and target membranes, which are designated as vesicle (v-) SNARE and target (t-) SNARE, respectively. $v$-SNARE consists of the vesicle-associated membrane protein (VAMP) family, while t-SNARE consists of the syntaxin family and the synaptosomal-associated protein of $25 \mathrm{kDa}$ (SNAP-25) family. v-SNAREs and their corresponding t-SNAREs recognize each other and form a stable trans-SNARE complex, which brings vesicles close to the acceptor membrane and mediates vesicle fusion. $\alpha$-Taxilin binds to syntaxin family members via their C-terminal coiled-coil region, which is involved in SNARE complex formation (26), but $\alpha$-taxilin is unable to interact with syntaxin- 1 after it forms a complex with VAMP2, SNAP-25, or Munc18 (27). Therefore, it has been postulated that $\alpha$-taxilin may be involved in tethering of vesicles to the acceptor membrane before formation of the trans-SNAREs complex.

\section{Transcription and translation}

On the other hand, it has been recently reported that $\alpha$-taxilin interacts with the nascent polypeptide-associated complex (NAC), mainly through its alphasubunit $(\alpha \mathrm{NAC})$ (44). There is increasing evidence

Table 1 Cox regression analysis for various potential prognostic factors in survival

\begin{tabular}{|c|c|c|c|c|c|c|c|c|c|c|c|}
\hline \multirow[b]{2}{*}{ Variable } & \multirow[b]{2}{*}{$\begin{array}{c}\text { Unfavorable/ } \\
\text { favorable } \\
\text { characteristics }\end{array}$} & \multicolumn{5}{|c|}{ Overall survival in all patients } & \multicolumn{5}{|c|}{ Disease-free survival in M0 patients at nephrectomy } \\
\hline & & $\begin{array}{c}\text { No. of } \\
\text { Patients }\end{array}$ & Analysis & $\begin{array}{c}\text { Relative } \\
\text { risk }\end{array}$ & $\begin{array}{c}95 \% \\
\text { confidential } \\
\text { interval } \\
\end{array}$ & $P$ value & $\begin{array}{l}\text { No. of } \\
\text { Patients }\end{array}$ & Analysis & $\begin{array}{l}\text { Relative } \\
\text { risk }\end{array}$ & $\begin{array}{c}95 \% \\
\text { confidential } \\
\text { interval } \\
\end{array}$ & $P$ value \\
\hline \multirow{2}{*}{ Grade } & \multirow{2}{*}{$3 / 2 / 1$} & \multirow{2}{*}{$12 / 33 / 7$} & Univariate $(\mathrm{U})$ & 2.796 & $0.821-\quad 3.525$ & 0.1601 & $6 / 27 / 4$ & $\mathrm{U}$ & 2.186 & $0.321-14.886$ & 0.4244 \\
\hline & & & Multivariate $(\mathrm{M})$ & 1605 & $0.583-4.419$ & 0.3599 & & M & 1.508 & $0.106-45.433$ & 0.7674 \\
\hline \multirow{2}{*}{$\mathrm{pT}$} & \multirow{2}{*}{$4,3 / 2,1$} & \multirow{2}{*}{$18 / 34$} & U & 3.282 & $0.814-13.256$ & 0.0947 & $9 / 28$ & $\mathrm{U}$ & 1.267 & $0.132-12.197$ & 0.8375 \\
\hline & & & M & 1.474 & $0.306-7.105$ & 0.6289 & & M & 1.696 & $0.122-28.233$ & 0.7126 \\
\hline \multirow{2}{*}{ PVI* } & \multirow{2}{*}{$(+) /(-)$} & \multirow{2}{*}{$20 / 32$} & $\mathrm{U}$ & 10.694 & $1.294-88.382$ & 0.0279 & $10 / 27$ & $\mathrm{U}$ & 7.198 & $0.671-77.180$ & 0.1629 \\
\hline & & & M & 4.273 & $0.305-59.868$ & 0.2809 & & M & 5.729 & $0.365-90.021$ & 0.2141 \\
\hline \multirow{2}{*}{$\mathrm{M}$} & \multirow{2}{*}{$(+) /(-)$} & \multirow{2}{*}{$15 / 37$} & $\mathrm{U}$ & 16.310 & $2.031-130.989$ & 0.0086 & & & & & \\
\hline & & & $\mathrm{M}$ & 7.795 & $0.626-97.123$ & 0.1105 & & & & & \\
\hline \multirow{2}{*}{ Taxilin } & \multirow{2}{*}{ high / low } & \multirow{2}{*}{$19 / 33$} & $\mathrm{U}$ & 4.733 & $0.933-24.002$ & 0.0605 & $9 / 28$ & $\mathrm{U}$ & 11.632 & $1.144-106.422$ & 0.0379 \\
\hline & & & $\mathrm{M}$ & 1.562 & $0.076-\quad 4.136$ & 0.5711 & & M & 6.004 & $0.468-77.072$ & 0.1686 \\
\hline
\end{tabular}

PVI*: Pathological vessel invasion 
that $\alpha$ NAC has a role in transcriptional processes (35), while NAC itself is associated with translation (4). Therefore, these findings suggest that the taxilin family could play an important role in the process of translation through interaction with NAC, as well as transcription through interaction with aNAC alone (44). Correct transmission of genetic information through both transcriptional and translational processes is important for normal cell function. Defects of this system may result in abnormal segregation of chromosomes and genomic instability, which are characteristic of many cancers (12). It has also been reported that $\alpha \mathrm{NAC}$ is expressed in malignant brain tumor cells (19). Papachristou et al. showed that $\alpha \mathrm{NAC}$ was expressed in osteosarcoma, but not in normal bone, and was expressed more strongly in high grade tumors compared with low grade tumors on immunohistochemical analysis (31). aNAC is involved in a coactivator potentiating c-Jun-mediated transcription process $(20,33,34)$. Oya et al. have reported that activation of C-Jun is acquired during aberrant proliferation of RCC (30). We previously reported that $\alpha$-taxilin mainly binds to NAC through its interaction with $\alpha \mathrm{NAC}$, indicating that $\alpha$-taxilin is involved in the processes of translation and transcription (44). Yu et al. recently found that the interaction of activating transcription factor (ATF) 4 with factor inhibiting ATF4-mediated transcription, also known as $\gamma$-taxilin (FIAT/ $\gamma$-taxilin), inhibits ATF4-mediated transcription (45). In addition, FIAT/ $\gamma$-taxilin interacts with the transcriptional coactivator aNAC (45), so we should study the role of mutual interactions of the taxilin family in cancer. A pathway that includes VHL-HIF-1 alpha-VEGF seems to be important for metastasis of clear cell RCC, and targeting this pathway may improve the outcome for patients with metastatic RCC (38).

Our study included a relatively small number of patients and the follow-up period was too short to draw definite conclusions regarding the possible relation between $\alpha$-taxilin and the effects of treatment or the prognosis. However, our finding that increased $\alpha$-taxilin expression is associated with invasion and metastasis of RCC suggests a possible role of deregulated $\alpha$-taxilin in the progression of human cancer and the potential availability of $\alpha$-taxilin as a target for treatment. Therefore, we should study the relationship between the effects of IFN- $\alpha$, sorafenib, or sunitinib and expression of $\alpha$-taxilin protein in order to examine the metastatic and invasive potential of $\alpha$-taxilin. Such information may shed light on the value of $\alpha$-taxilin as a biomarker.

\section{Acknowledgements}

The authors are grateful to Hitomi Yamazaki for her excellent technical assistance, and Kazumoto Kimura, $\mathrm{PhD}$, for his constructive suggestions regarding statistical analysis in this study.

\section{REFERENCES}

1. Athar U and Gentile TC (2008) Treatment options for metastatic renal cell carcinoma: a review. Can J Urol 15, 523532.

2. Bascom JL, Fata JE, Hirai Y, Sternlicht MD and Bissell MJ (2005) Epimorphin overexpression in the mouse mammary gland promotes alveolar hyperplasia and mammary adenocarcinoma. Cancer Res 65, 8617-8621.

3. Bergers G, Brekken R, McMahon G, Vu TH, Itoh T, Tamaki K, Tanzawa K, Thorpe P, Itohara S, Werb Z and Hanahan D (2000) Matrix metalloproteinase-9 trigers the angiogenic switch during carcinogenesis. Nat Cell Biol 2, 737-744.

4. Braat AK, Yan N, Arn E, Harrison D and Macdonald PM (2004) Localization-dependent Oskar protein accumulation; control after the initiation of translation. Dev Cell 7, 125131.

5. Coppin $C$ (2008) Immunotherapy for renal cell cancer in the era of targeted therapy. Expert Rev Anticancer Ther 8, 97103.

6. Etienne-Manneville S and Hall A (2002) Rho GTPases in cell biology. Nature 420, 629-635.

7. Fritz G, Just I and Kaina B (1999) Rho GTPase overexpressed in human tumors. Int $J$ Cancer 81, 682-687.

8. Fuhrman SA, Lasky LC, Lmas C, Motzer RJ, Russo P, Nanus DM and Berg WJ (1982) Renal cell carcinoma. Am J Surg Pathol 6, 655-663.

9. Griedl P and Wolf K (2003) Tumour-cell invasion and migration: diversity and escape mechanisms. Nat Rev Cancer 3, 362-374.

10. Jahn R, Lang T and Sudhof TC (2003) Membrane fusion. Cell 112, 519-533.

11. Jahn R and Scheller RH (2006) SNAREs-engines for membrane fusion. Nat Rev Mol Cell Biol 7, 631-643.

12. Jallepalli PV and Lengauer C (2001) Chromosome segregation and cancer: cutting through the mystery. Nat Rev Cancer 1, 109-117.

13. Jantzer P and Schendel DJ (1998) Human renal carcinoma antigen-specific CTLs, antigen-driven selection and long-term persistence in vivo. Cancer Res 58, 3078-3086.

14. Jones MC, Caswell PT and Norman JC (2006) Endocytic recycling pathways: emerging regulators of cell migration. Curr Opin Cell Biol 18, 549-557.

15. Kamai T, Tsujii T, Arai K, Takagi K, Asami H, Ito Y, Oshima $\mathrm{H}$ and Yoshida K-I (2003) Significant association of Rho/ ROCK pathway with invasion and metastasis of bladder cancer. Clin Cancer Res 9, 2632-2641.

16. Kamai T, Shirataki H, Nakanishi K, Furuya N, Kambara T, Abe H, Oyama T and Yoshida K-I (2010) Increased Rac1 activity and Pak1 overexpression are associated with lymphovascular invasion and lymph node metastasis of upper urinary tract cancer. BMC Cancer 10, 1471-2407-10-164.

17. Kallakury BV, Karikehalli S, Haholu A, Sheehan CE, Azumi $\mathrm{N}$ and Ross JS (2001) Increased expression of matrix metalloproteinases 2 and 9 and tissue inhibitors of metalloproteinases 1 and 2 correlate with poor prognostic variables in renal 
cell carcinoma. Clin Cancer Res 7, 3113-3119.

18. Kean MJ, Williams KC, Skalski M, Myers D, Burtnik A, Foster D and Coppolino MG (2009) VAMP3, syntaxin-13 and SNAP23 are involved in secretion of matrix metalloproteinases, degradation of the extracellular matrix and cell invasion. J Cell Sci 122, 4089-4098.

19. Kroes RA, Jastrow A, McLone MG, Yamamoto H, Colley P, Kersey DS, Yong VW, Mkrdichian E, Cerullo L, Leestma J and Moskal J (2000) The identification of novel therapeutic targets for the treatment of malignant brain tumors. Cancer Lett 156, 191-198.

20. Moreau A, Yotov WV, Glorieux FH and St-Arnaud R (1998) Bone-specific expression of the alpha chain of the nascent polypeptide-associated complex, a coactivator potentiating c-Jun-mediated transcription. Mol Cell Biol 18, 1312-1321.

21. Linehan WM, Walther MM and Zbar B (2003) The genetic basis of cancer of the kidney. $J$ Urol 170, 2163-2172.

22. Miyata T, Ohnishi H, Suzuki J, Yoshikumi Y, Ohno H, Mashima H, Yasuda H, Ishijima T, Osawa H, Satoh K, Sunada K, Kita H, Yamamoto H and Sukuno K (2004) Involvement of syntaxin 4 in the transport of membrane-type 1 matrix metalloproteinase to the plasma membrane in human gastric epithelial cells. Biochem Biophys Res Commun 323, 118124.

23. Motzer RJ, Bander NH and Nanus DM (1996) Renal-cell carcinoma. $N$ Engl J Med 335, 865-875.

24. Nabi IR (1999) The polarization of the motile cell. J Cell Sci 112, 1803-1811.

25. Nakanishi K, Kamai T, Mizuno T, Arai K and Yamanishi T (2009) Expression of RhoA mRNA and activated RhoA in urothelium and smooth muscle, and effects of a Rho-kinase inhibitor on contraction of the porcine urinary bladder. Neurourol Urodynam 28, 521-528.

26. Nogami S, Satoh S, Nakano M, Shimizu H, Fukushima H, Maruyama A, Terano A and Shirataki H (2003) Taxilin: a novel syntaxin-binding protein that is involved in $\mathrm{Ca}^{2+}$. dependent exocytosis in neuroendocrine cells. Genes Cells $\mathbf{8}$, $17-28$.

27. Nogami S, Satoh S, Nakano M, Terano A and Shirataki H (2003) Interaction of taxilin with syntaxin which does not form the SNARE complex. Biochem Biophys Res Commun 311, 797-802.

28. Nogami S, Satoh S, Tanaka-Nakadate S, Yoshida K, Nakano M, Terano A and Shirataki H (2004) Identification and characterization of taxilin isoforms. Biochem Biophys Res Commun 319, 936-943.

29. Oba-Shinjo SM, Benqtson MH, Winnisvhofer SM, Colin C, Vedoy CG, de Mendonca A, Marie SK and Soqayar MC (2005) Identification of novel differentially expressed genes in human astrocytomas by cDNA representational difference analysis. Brain Res Mol Brain Res 140, 25-33.

30. Oya M, Mikami S, Mizuno R, Marumo K, Mukai M and Murai M (2005) C-jun activation in acquired cyctic kidney disease and renal cell carcinoma. J Urol 174, 726-730.
31. Papachristou DJ, Batistatou A, Sykiotis GP, Varakis I and Papavassiliou AG (2003) Activation of the JNK-AP-1 signal transduction pathway is associated with pathogenesis and progression of human osteosarcomas. Bone 32, 364-371.

32. Quigley JP and Armstrong PB (1998) Tumor cell intravasation alu-cidated; The chick embryo opens the window. Cell 94, 281-284.

33. Quelo I, Akhouayri O, Prud homme J and St-Arnaud R (2004) GSK3b-dependent phosphorylation of the aNAC coactivator regulates its nuclear translocation and proteasome-mediated degradation. Biochemistry 43, 2906-2914.

34. Quelo I, Gauthier C, Hannigan GE, Dedhar S and St-Arnaud $R$ (2004) Integrin-linked kinase regulates the nuclear entry of the c-Jun coactivator $\alpha$-NAC and its coactivation potency. $J$ Biol Chem 279, 43893-43899.

35. Rospert S, Dubaquie Y and Gautschi M (2002) Nascent-polypeptide-associated complex. Cell Mol Life Sci 59, 16321639.

36. Sahai E and Marshall CJ (2002) Rho-GTPases and cancer. Nat Rev Cancer 2, 133-142.

37. Sakakibara S, Nakadate K, Tanaka-Nakadate S, Yoshida K, Nogami S, Shirataki H and Ueda S (2008) Developmental and spatial expression pattern of alpha-taxilin in the rat central nervous system. $J$ Comp Neurol 511, 65-80.

38. Sciarra A, Gentile V, Salciccia S, Alfarone A and Di Silverio F (2008) New anti-angiogenic targeted therapy in advanced renal cell carcinoma (RCC): current status and future prospects. Rev Recent Clin Trials 3, 97-103.

39. Sobin $\mathrm{H}$ and Wittekind $\mathrm{CH}$, eds. (2002) International union against cancer. UICC, In TNM Classification of Malignant Tumors, 6rd ed. Wiley-Liss, New York.

40. Stetler-Stevenson WG, Liotta LA and Kleiner DE Jr (1993) Extracellular matrix 6: role of matrix metalloproteinases in tumor invasion and metastasis. FASEB $J$ 7, 1434-1441.

41. Suwa H, Ohshio G, Imamura T, Watanabe G, Arii S, Imamura M, Narumiya S, Hiai H and Fukumoto M (1998) Overexpression of the $r h o \mathrm{C}$ gene correlates with progression of ductal adenocarcinoma of the pancreas. Br $J$ Cancer 77, 147152.

42. Tayeb MA, Skalski M, Cha MC, Kean MJ, Scaife M and Coppolino MG (2005) Inhibition of SNARE-mediated membrane traffic impairs cell migration. Exp Cell Res 305, 6373.

43. Wright PK (2008) Targeting vesicle trafficking: An important approach to cancer chemotherapy. Recent Pat Anticancer Drug Discov 3, 137-147.

44. Yoshida K, Nogami S, Satoh S, Tanaka-Nakadate S, Hiraishi $\mathrm{H}$, Terano A and Shirataki H (2005) Interaction of the taxilin family with the nascent polypeptide-associated complex that is involved in the transcriptional and translational processes. Genes Cells 10, 465-476.

45. Yu VW, Gauthier C and St-Arnaud R (2006) Inhibition of ATF4 transcriptional activity by FIAT/gamma-taxilin modulates bone mass accrual. Ann N Y Acad Sci 1068, 131-142. 